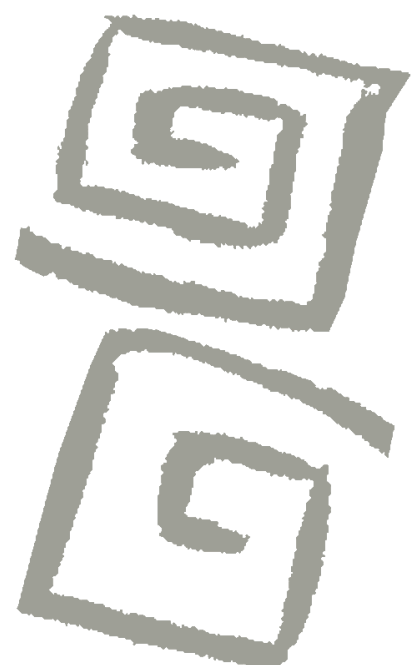

\title{
Muertes ocultas: estimaciones corregidas de homicidios en Bahía, Brasil, 1996-2015
}

\author{
Hidden deaths: corrected estimates of homicides in \\ Bahia, Brazil, 1996- 2015
}

Tiago Oliveira de Souza ${ }^{1}$, Liana Wernersbach Pinto ${ }^{2}$, Edinilsa Ramos de Souza ${ }^{3}$

'Enfermero. Doctor en Salud Pública. Profesor, Enfermería y Obstetricia, Universidade Federal do Rio de Janeiro, Brasil. $\bowtie$ iD

${ }^{2}$ Nutricionista, Doctora en Ingeniería Biomédica. Investigadora, Departamento de Estudos sobre Violencia e Salud Jorge Careli, Fundação Oswaldo Cruz, Rio de Janeiro, Brasil. $\square$ (iD

${ }^{3}$ Psicóloga, Doctora en Salud Pública. Investigadora, Departamento de Estudos sobre Violencia e Salud Jorge Careli, Fundação Oswaldo Cruz, Rio de Janeiro, Brasil. $\triangle$ id
RESUMEN El objetivo del estudio fue corregir y estimar las tasas quinquenales de homicidios en Bahía, Brasil, en el período 1996-2015. Se realizó un estudio ecológico de los homicidios a partir de datos oficiales del Sistema de Información sobre Mortalidad del Ministerio de Salud. Estas defunciones por agresiones corresponden a los códigos X85-Y09 de la Clasificación internacional de enfermedades $10^{\mathrm{a}}$ revisión (CIE 10). Se realizó una redistribución proporcional de las defunciones clasificadas como eventos de intención indeterminada (Y10-Y34) y se aplicaron factores de corrección de la mortalidad. En el período analizado, se registraron 67.599 homicidios en el Sistema de Información sobre Mortalidad y, luego de la segunda corrección, se estimaron 88.429 homicidios. Al comparar los números oficiales a los ajustados, se observó una subnotificación del 30,8\%. Las mayores tasas de homicidio corregidas se observaron en la región este, en Pojuca (129,8 homicidios por 100.000 habitantes), Lauro de Freitas $(117,7)$ y Simões Filho $(114,3)$; en la región sur, en Santa Luzia $(121,4)$, Valença $(87,6)$ e Itabuna $(86,5)$; y en el extremo sur, en Santa Cruz Cabrália $(128,2)$, Itabela $(113,3)$ y Porto Seguro $(106,8)$. Luego de las correcciones, se observó un incremento de los homicidios en todos los quinquenios en los municipios analizados, lo que resultó en una tasa de mortalidad aún más elevada.

PALABRAS CLAVES Homicidio; Mortalidad; Técnicas de Estimación; Sistemas de Información en Salud; Distribución Temporal; Brasil.

ABSTRACT The objective of this study was to correct and estimate five-year homicide rates in Bahia, Brazil, for the 1996-2015 period. An ecological study of the homicides was carried out using official data from the Mortality Information System of the Ministry of Health. Deaths due to assault are classified in the codes X85-Y09 of the International Classification of Disease 10th Revision (ICD-10). A proportional redistribution of deaths classified as events of undetermined intent (Y10-Y34) was carried out and mortality correction factors were applied. In the analyzed period, 67,599 homicides were registered in the Mortality Information System; after the second correction, 88,429 homicide deaths were estimated. Comparing the official and adjusted figures, there was an underreporting of $30.8 \%$. The highest corrected homicide rates were observed in the eastern region, in Pojuca (129.8 homicides per 100,000 inhabitants), Lauro de Freitas (117.7) and Simões Filho (114.3); in the southern region, in Santa Luzia (121.4), Valença (87.6) and Itabuna (86.5); and in the far south, Santa Cruz Cabrália (128.2), Itabela (113.3) and Porto Seguro (106.8). After correction, there was an increase in homicides in all of the five-year periods in the municipalities analyzed, which resulted in an even higher mortality rate.

KEY WORDS Homicide; Mortality; Estimation Techniques; Health Information Systems; Temporal Distribution; Brazil. 


\section{INTRODUCCIÓN}

El homicidio, como expresión de violencia interpersonal y causa específica de defunciones, ha sido ampliamente discutido en el área de la salud colectiva por tratarse de un importante indicador social y de salud. Sus impactos, su magnitud y sus consecuencias directas e indirectas, individuales y colectivas, micro y macro sociales, han sido destacadas en debates y publicaciones locales e internacionales ${ }^{(1)}$.

De acuerdo con el informe de la Organización Mundial de la Salud (OMS), presentado en 2014, el 10\% de los homicidios ocurridos en el mundo se cometen en Brasil(2). Datos nacionales ${ }^{(3)}$ señalan que, en 2015 , en Brasil, ocurrieron 58.138 homicidios, de los cuales el 39,6\% (22.999 casos) corresponde a la región nordeste. En el mismo año, el estado de Bahía registró el mayor número absoluto de homicidios (5.787) en Brasil, el cual representó el $25,2 \%$ de los homicidios de la región nordeste, superando en números absolutos, a estados más poblados como São Paulo (5.150 homicidios) y Río de Janeiro (4.786 homicidios).

Algunas décadas antes, estos mismos registros oficiales mostraban otra realidad ${ }^{(3)}$. En 1996, el estado bahiano presentaba un total de 1.883 muertes por homicidio, número bastante inferior a los de los estados que actualmente supera: en aquel año, São Paulo informó 12.320 casos, Río de Janeiro y Pernambuco registraron 8.030 y 3.022 homicidios, respectivamente.

De acuerdo con el Mapa de la violencia $2014^{(4)}$, entre los años 1998 y 2012, Bahía pasó de la posición 22 a la 5 en el orden de las unidades federativas por tasas de homicidio (por 100.000 habitantes), en la población total. En el período de 14 años, la tasa de homicidio en ese estado pasó de 9,7/100.000 habitantes en 1998 ( 1.271 casos), a 41,9/100.000 habitantes en 2012 (5.936 casos).

Una publicación del Ministerio de Justicia señaló a Bahía como el estado con la mayor concentración en números absolutos de homicidios en 2014. La información destaca que Bahía registró, en ese año, 5.450 homicidios, lo que representa más del 10\% de todos los casos de homicidio entre las unidades federativas. Señala, además, que Bahía también se destaca por su elevada tasa de homicidios por 100.000 habitantes, la sexta mayor del país. Según el Pacto Nacional por la Reducción de Homicidios (PNRH) del Ministerio de Justicia, citado en el mismo documento, Bahía presenta el mayor número de municipios incluidos en ese Pacto (diez municipios), que comprende un total de 81 ciudades del país ${ }^{(5)}$.

Sobre el perfil epidemiológico de causas externas, el Ministerio de Salud ya señalaba en el año 2010, que los jóvenes y adultos jóvenes, en la franja etaria de los 15 a los 39 años de edad, de sexo masculino, residentes en la región nordeste, eran los más vulnerables a la muerte por homicidio, en las regiones del país. Los homicidios representaron el $36,8 \%$ del total de defunciones por causas externas en el año 2009, y se destacaron como la primera causa de muerte en la franja etaria citada ${ }^{(6)}$.

Por otro lado, en el ranking de calidad de la información sobre mortalidad violenta en las unidades federativas, entre 2007 y 2010, Bahía presentó la segunda mayor tasa de muerte con intención indeterminada del país. Además, ese estado tiene la mayor diferencia entre el crecimiento de las tasas de homicidios oficiales y estimadas/corregidas en el período de 1996-2010 ${ }^{(7)}$. Esto significa que una parte considerable de los homicidios que ocurren en Brasil, y más específicamente, en el estado de Bahía, no son contabilizados por las estadísticas oficiales y una gran proporción de los que son captados por las estadísticas vitales, no pueden ser clasificados como homicidios, dado que se desconoce la intención que llevó a una muerte violenta.

En lo que se refiere a la causa básica de la defunción, el elevado número de muertes clasificadas como causas externas cuya intención es indeterminada, se torna un obstáculo para la construcción de indicadores de mortalidad más fidedignos y tiene un peso importante y limitante en las inferencias realizadas sobre los indicadores de salud ${ }^{(8)}$. En otras 
palabras, no se puede avanzar sobre el perfil de homicidios, variables caracterizadoras de las defunciones y de las víctimas u otras variables de estudio de la mortalidad, sin la comprensión de la limitación causada por la subnotificación de defunciones en lo que se refiere a la clasificación/contabilización de la causa básica de defunción, sobre todo, en las causas violentas como los homicidios.

Sobre ese grupo de muertes, se sabe que derivan de una lesión (por lo tanto, una causa externa), pero sin clarificar la intencionalidad, por lo que recibe la denominación de eventos de intención indeterminada ${ }^{(9)}$. La mayor participación de este grupo en el conjunto de las causas externas expresa un menor esclarecimiento de la causa básica de defunción y una disminución de la clasificación adecuada de las defunciones en los grupos específicos de causas externas (como homicidio, suicidio y accidente) cuya intencionalidad es identificada.

De este modo, las fallas en el proceso de clasificación o elucidación de la información referente a la causa básica, distorsiona y subestima determinados resultados, que pueden alterar los perfiles epidemiológicos referentes a un tipo de causa de defunción específica. Por lo tanto, el análisis de datos a partir de los sistemas de información, sin algún tipo de corrección, en algunos casos, puede enmascarar la realidad y disminuir la confiabilidad de los hallazgos ${ }^{(10)}$.

Con relación al problema de la causa de la muerte, en los estudios de mortalidad, la indeterminación de la causa de muerte es un obstáculo importante, de tal modo que puede alterar, en forma significativa, la tasa de mortalidad por causa específica. Esto perjudicaría delinear el perfil epidemiológico de homicidios, por ejemplo, con la consecuente barrera para "pensar" y "aplicar" estrategias para enfrentarlo, y asignar recursos y políticas. Considerar, por lo tanto, las limitaciones derivadas de la indeterminación de causas de defunciones es extremadamente importante ${ }^{(11)}$.

Por eso, para determinar la causa básica de muerte, de tratarse de homicidio, es preciso considerar los medios utilizados en la agresión fatal, además de las lesiones, de los traumas y de las heridas derivadas de esa acción infligida. Siempre que la causa de muerte sea una agresión infligida por otra persona se debe seleccionar la causa básica de acuerdo con la codificación pertinente. Por lo tanto, para viabilizar estrategias de prevención no alcanza con conocer la naturaleza de las lesiones, por ejemplo, traumatismo craneano, herida por arma de fuego, y otros con intencionalidad indeterminada ${ }^{(11)}$, sino que es imprescindible conocer los tipos de agresiones (con disparo de arma corta; con disparo de rifle, escopeta o arma larga; con objeto cortante).

Ante esta situación, el presente estudio tuvo como objetivo estimar las tasas de homicidio quinquenales corregidas, por medio de la redistribución proporcional de defunciones y la aplicación de factores de corrección, con la finalidad de presentar indicadores de mortalidad más fidedignos, en Bahía, en el período 1996-2015.

\section{MATERIALES Y MÉTODOS}

Se realizó un estudio ecológico de defunciones por homicidio en residentes en el estado de Bahía, a partir de los datos del Sistema de Información sobre Mortalidad (SIM) del Departamento de Informática del Sistema Único de Salud (DATASUS) del Ministerio de Salud(3), referentes al período 1996-2015.

Las defunciones se agruparon en cuatro quinquenios para reducir la fluctuación aleatoria de los indicadores calculados y la inestabilidad en el análisis por municipio, al considerar recortes temporales y espaciales restringidos $^{(10)}$. Los períodos quinquenales se presentan en los resultados de la siguiente forma: 1996 a 2000 (Q1), 2001 a 2005 (Q2), 2006 a 2010 (Q3), 2011 a 2015 (Q4).

Se incluyeron los casos de homicidio (agresiones), clasificados en los códigos X85 a Y09 de la Clasificación Internacional de Enfermedades $10^{\text {a }}$ Revisión (CIE-10), referentes a la población residente en los 417 municipios del estado de Bahía, de acuerdo con las estimaciones poblacionales del Instituto Brasileño de 
Geografía y Estadística (IBGE), que componen los denominadores de las tasas de mortalidad, en el período de estudio ${ }^{(9,12)}$. Para el análisis, los municipios se agruparon en las nueve macrorregiones de salud que, a los efectos del proceso de regionalización de la asistencia a la salud, se delimitan según características demográficas y socioeconómicas: centro-este, centro-norte, extremo sur, este, nordeste, norte, oeste, sudoeste y sur.

Considerando que Bahía es uno de los siete estados del país con el mayor número de muertes violentas cuya intención no fue determinada, se realizó la redistribución proporcional de las defunciones clasificadas en ese grupo de eventos (códigos Y10-Y34) y luego se aplicaron factores de corrección de las tasas de mortalidad.

Esta redistribución fue hecha para los demás grupos de causas externas: accidentes de transporte (V01-V99); otras causas externas de traumatismos accidentales (W00-X59); lesiones autoinfligidas intencionalmente (X60$\mathrm{X} 84$ ); agresiones (X85-Y09); intervenciones legales (Y35) y complicaciones de la atención médica y quirúrgica (Y40-Y89). Comprendió las siguientes fases: 1) cálculo de la proporción de defunciones por agresiones (y de los demás grupos de causas) respecto del total de defunciones por causas externas con intención determinada (excluyendo las causas indeterminadas); 2) multiplicación de esa proporción por el número de defunciones con intención indeterminada, de lo que se obtiene la parte proporcional de defunciones por agresiones respecto de las defunciones por causa indeterminada; 3) suma de la parte proporcional con el número oficial de defunciones por agresiones registrados en el SIM. El resultado de esos cálculos corresponde al número de homicidios de la primera etapa de corrección.

Luego, a los homicidios corregidos en la primera etapa, se aplicaron (multiplicados) factores de corrección, según el estudio de Szwarcwald et al.(13), que estimó factores de corrección de la mortalidad para municipios, estados y macrorregiones de Brasil. Después de ese procedimiento, se obtuvo el número de defunciones resultante de la segunda etapa de corrección.
Entre los factores de corrección utilizados se consideró el tamaño poblacional de los municipios referente al año 2010, cuyas categorías también siguieron la clasificación del estudio de Szwarcwald et al. ${ }^{(13)}$ : hasta 20.000 habitantes (pequeño); 20.001 a 50.000 habitantes (pequeño/medio); 50.001 a 200.000 habitantes (medio/grande); más de 200.000 habitantes (grande).

Se calcularon las tasas de homicidios para cada año del período estudiado. Se utilizó como numerador el número de defunciones corregidas en la segunda etapa, o sea, luego de la multiplicación por los factores de corrección y, como denominador, la población residente, referente a cada año del estudio. De este modo, se obtuvo la tasa de homicidios corregida. Posteriormente, se calculó la tasa media de homicidios por 100.000 habitantes para cada quinquenio, a través de una media aritmética simple. En la presentación de los resultados se ordenaron los 20 municipios con mayores tasas de homicidio de acuerdo con el último quinquenio (Q4), que comprende el período 2011-2015. A continuación, en cada una de las macrorregiones de salud se ordenaron los tres municipios con las mayores tasas, referentes al último quinquenio.

Los programas utilizados en la tabulación de los datos, la construcción del banco y el cálculo de las tasas, estimaciones y correcciones fueron, inicialmente, TabNet y TabWin, y luego los datos se consolidaron en Excel $^{\odot}$ y se procesaron en Statistical Package for Social Science (SPSS ${ }^{\odot}$ ) versión 23. Se utilizaron solo datos de dominio público y de acceso irrestricto, sin identificación de individuos, en conformidad con la Resolución 466/2012 del Consejo Nacional de Salud del Ministerio de Salud.

\section{RESULTADOS}

En Bahía, en el período de 1996-2015, el SIM registró oficialmente 67.599 homicidios, con una tasa bruta de 24,4 homicidios por 100.000 habitantes. En la primera 
corrección, luego de la redistribución proporcional de las defunciones de intencionalidad indeterminada, ese total subió a 78.948 homicidios.

En la segunda corrección, al aplicar los factores, se estimaron 88.429 homicidios (Tabla 1), con una tasa de homicidios corregida de 31,1 defunciones por 100.000 habitantes, en el último quinquenio (Q4) (Tabla 2). Al comparar el número de defunciones oficiales con el de la segunda corrección, se observó una subnotificación del 30,8\% (una diferencia de 20.830 defunciones) en los datos obtenidos directamente del SIM, en todos los municipios del estado y en todo el período analizado.

Al considerar los quinquenios, el segundo (Q2), en el período 2001-2005, presentó la mayor subestimación de defunciones $(50,2 \%)$, con una diferencia de más de 5.000 homicidios entre los datos oficiales (SIM) y el número de homicidios estimados en la segunda corrección.

Tabla 1. Número absoluto y corregido de homicidios por quinquenio, en los 20 municipios con mayores tasas de homicidio. Estado de Bahía, Brasil, 1996-2015.

\begin{tabular}{|c|c|c|c|c|c|c|c|c|c|c|c|c|c|}
\hline \multirow{3}{*}{ Estado/Municipios } & \multicolumn{12}{|c|}{ Número de homicidios } & \multirow{3}{*}{$\begin{array}{l}\text { Factor de } \\
\text { corrección }^{d}\end{array}$} \\
\hline & \multicolumn{4}{|c|}{$\mathrm{SIM}^{\mathrm{a}}$} & \multicolumn{4}{|c|}{ Primera corrección } & \multicolumn{4}{|c|}{ Segunda corrección ${ }^{c}$} & \\
\hline & Q1 & Q2 & Q3 & Q4 & Q1 & Q2 & Q3 & Q4 & Q1 & Q2 & Q3 & Q4 & \\
\hline Bahía & 7.071 & 10.464 & 22.179 & 27.885 & 7.932 & 14.106 & 25.878 & 31.032 & 8.868 & 15.714 & 28.864 & 34.983 & - \\
\hline Pojuca & 13 & 17 & 44 & 186 & 16 & 23 & 51 & 193 & 20 & 27 & 61 & 233 & 1,21 \\
\hline Santa Cruz Cabrália & 8 & 15 & 51 & 147 & 8 & 17 & 55 & 147 & 10 & 21 & 67 & 178 & 1,21 \\
\hline Santa Luzia & 4 & 13 & 41 & 59 & 4 & 15 & 44 & 67 & 5 & 18 & 54 & 82 & 1,22 \\
\hline Lauro de Freitas & 172 & 183 & 627 & 879 & 202 & 280 & 746 & 947 & 226 & 314 & 835 & 1061 & 1,12 \\
\hline Simões Filho & 64 & 155 & 442 & 592 & 69 & 234 & 527 & 647 & 77 & 262 & 590 & 725 & 1,12 \\
\hline Itabela & 36 & 49 & 69 & 123 & 39 & 53 & 75 & 142 & 47 & 64 & 91 & 172 & 1,21 \\
\hline Mata de São João & 22 & 36 & 57 & 185 & 25 & 48 & 69 & 197 & 30 & 58 & 84 & 239 & 1,21 \\
\hline Porto Seguro & 103 & 211 & 526 & 645 & 110 & 225 & 562 & 656 & 123 & 252 & 629 & 735 & 1,12 \\
\hline Eunápolis & 109 & 161 & 410 & 414 & 115 & 175 & 440 & 480 & 129 & 196 & 493 & 538 & 1,12 \\
\hline Valença & 56 & 54 & 166 & 358 & 58 & 61 & 184 & 367 & 65 & 69 & 206 & 411 & 1,12 \\
\hline Teixeira de Freitas & 101 & 120 & 345 & 517 & 111 & 164 & 420 & 578 & 124 & 184 & 470 & 648 & 1,12 \\
\hline Itabuna & 246 & 442 & 794 & 816 & 261 & 499 & 837 & 859 & 280 & 534 & 895 & 919 & 1,07 \\
\hline Uruçuca & 28 & 30 & 57 & 69 & 32 & 39 & 61 & 72 & 39 & 47 & 74 & 88 & 1,22 \\
\hline Amélia Rodrigues & 8 & 6 & 43 & 80 & 8 & 10 & 55 & 89 & 10 & 12 & 67 & 108 & 1,21 \\
\hline Dias d'Ávila & 32 & 52 & 205 & 258 & 38 & 76 & 245 & 273 & 42 & 85 & 274 & 306 & 1,12 \\
\hline Itapebi & 12 & 17 & 23 & 33 & 13 & 18 & 28 & 37 & 15 & 22 & 34 & 45 & 1,22 \\
\hline Vera Cruz & 14 & 10 & 83 & 133 & 17 & 13 & 91 & 140 & 21 & 15 & 110 & 169 & 1,21 \\
\hline Barro Preto & 4 & 2 & 11 & 21 & 4 & 2 & 11 & 21 & 5 & 2 & 13 & 26 & 1,22 \\
\hline Candeias & 55 & 98 & 199 & 274 & 64 & 150 & 250 & 305 & 71 & 169 & 280 & 342 & 1,12 \\
\hline Camaçari & 135 & 284 & 590 & 929 & 160 & 412 & 714 & 995 & 171 & 440 & 763 & 1064 & 1,07 \\
\hline \multicolumn{14}{|c|}{$\begin{array}{l}\text { Fuente: Elaboración propia a partir de datos del Sistema de Información sobre Mortalidad del Departamento de Informática del Sistema Único de Salud (SIM/ } \\
\text { DATASUS). } \\
\text { aSumatoria del número de homicidios quinquenales, obtenido directamente del SIM, sin corrección. } \\
\text { "Número de homicidios corregido mediante el cálculo de la proporción de defunciones por agresiones en relación con el total de defunciones por causas externas, } \\
\text { multiplicado por el número de defunciones de intención indeterminada, sumados al número oficial de defunciones por agresiones registrados en el SIM, lo que } \\
\text { da como resultado el número de defunciones por homicidio de la primera etapa de corrección. } \\
\text { "Número de homicidios obtenido en la primera etapa de corrección, multiplicado por los factores de corrección, considerando el tamaño poblacional de los } \\
\text { municipios en el año } 2010 \text {. }\end{array}$} \\
\hline
\end{tabular}


Al ordenar los 20 municipios por las tasas corregidas de homicidio más elevadas en el último quinquenio (Q4), en el período 2011-2015, se verifica que estas se encuentran en las macrorregiones de salud este, extremo-sur y sur (Tabla 2). Los tres primeros municipios con altas tasas en cada una de esas regiones fueron: en la macrorregión este, los municipios de Pojuca (129,8 homicidios por 100.000 habitantes), Lauro de Freitas $(117,7 / 100.000)$ y Simões Filho (114,3/100.000); en la región sur, Santa Luzia $(121,4 / 100.000)$, Valença $(87,6 / 100.000)$ y Itabuna (86,5/100.000); y en el extremo sur, los municipios de Santa Cruz Cabrália (128,2/100.000), Itabela (113,3/100.000) y Porto Seguro (106,8/100.000). Entre los 20 municipios destacados en la Tabla 2, solo Amélia Rodrigues forma parte de una macrorregión de salud distinta de las ya mencionadas. Este municipio pertenece a la región centro-este, y su tasa de homicidio corregida quedó en 83,7/100.000 habitantes.

Otro hallazgo es que algunos municipios aumentaron enormemente sus tasas entre el primer quinquenio (Q1) y el último (Q4), municipios de menor tamaño como Santa Cruz Cabrália (en los que la tasa osciló entre 10,1 y 128,2 homicidios por 100.000 habitantes) y Santa Luzia (que osciló entre 6,6 y 121,4 por 100.000 habitantes) tuvieron un aumento de más de $1.000 \%$ entre Q1 y Q4. Municipios mayores, como Simões Filho, también presentaron un incremento en el orden de más del $400 \%$, quintuplicando su tasa de homicidio entre el período quinquenal inicial y el final, varió del 19,2/100.000 en Q1, a 114,3/100.000 en Q4 (Tabla 2).

Tabla 2. Tasa de homicidios corregida, por quinquenio, macrorregión y tamaño poblacional, en los 20 municipios con mayores tasas de homicidio. Estado de Bahía, Brasil, 1996-2015.

\begin{tabular}{|c|c|c|c|c|c|c|}
\hline \multirow[t]{2}{*}{ Estado / municipios } & \multirow[t]{2}{*}{ Macrorregión } & \multirow[t]{2}{*}{ Tamaño poblacional } & \multicolumn{4}{|c|}{ Tasa de homicidios corregida ${ }^{a}$} \\
\hline & & & Q1 & Q2 & Q3 & $\mathrm{Q} 4^{\mathrm{b}}$ \\
\hline Bahía & - & - & 7,4 & 12,2 & 21,7 & 31,1 \\
\hline Pojuca & Este & Pequeño/medio & 16,1 & 19,9 & 38,8 & 129,8 \\
\hline Santa Cruz Cabrália & Extremo-sur & Pequeño/medio & 10,1 & 13,6 & 44,4 & 128,2 \\
\hline Santa Luzia & Sur & Pequeño & 6,6 & 23,6 & 73,1 & 121,4 \\
\hline Lauro de Freitas & Este & Medio/grande & 43,8 & 48,5 & 107,6 & 117,7 \\
\hline Simões Filho & Este & Medio/grande & 19,2 & 51,6 & 102,7 & 114,3 \\
\hline Itabela & Extremo-sur & Pequeño/medio & 34,0 & 46,6 & 64,1 & 113,3 \\
\hline Mata de São João & Este & Pequeño/medio & 18,5 & 34,8 & 43,7 & 110,6 \\
\hline Porto Seguro & Extremo-sur & Medio/grande & 33,3 & 41,9 & 97,0 & 106,8 \\
\hline Eunápolis & Extremo-sur & Medio/grande & 28,9 & 44,1 & 100,7 & 100,1 \\
\hline Valença & Sur & Medio/grande & 16,4 & 17,1 & 46,7 & 87,6 \\
\hline Teixeira de Freitas & Extremo-sur & Medio/grande & 24,1 & 31,8 & 73,4 & 86,6 \\
\hline Itabuna & Sur & Grande & 30,1 & 53,2 & 85,8 & 86,5 \\
\hline Uruçuca & Sur & Pequeño & 34,5 & 58,2 & 86,9 & 84,2 \\
\hline Amélia Rodrigues & Centro-este & Pequeño/medio & 8,3 & 9,7 & 54,6 & 83,7 \\
\hline Dias d'Ávila & Este & Medio/grande & 20,7 & 34,0 & 92,9 & 83,4 \\
\hline Itapebi & Extremo-sur & Pequeño & 27,3 & 39,4 & 60,5 & 83,4 \\
\hline Vera Cruz & Este & Pequeño/medio & 14,1 & 9,2 & 59,8 & 83,3 \\
\hline Barro Preto & Sur & Pequeño & 14,9 & 6,1 & 40,0 & 80,3 \\
\hline Candeias & Este & Medio/grande & 20,2 & 42,2 & 67,8 & 78,9 \\
\hline Camaçari & Este & Grande & 23,6 & 49,1 & 68,8 & 78,9 \\
\hline \multicolumn{7}{|c|}{$\begin{array}{l}\text { Fuente: Elaboración propia a partir de datos del Sistema de Información sobre Mortalidad del Departamento de } \\
\text { Informática del Sistema Único de Salud (SIM/DATASUS). } \\
\text { aCálculo de la tasa de homicidio quinquenal, cuyo numerador es el número de homicidios corregido y multiplicado } \\
\text { por los factores de corrección (por } 100.000 \text { habitantes), segunda corrección. } \\
\text { bLos períodos quinquenales } 1996-2000 \text { (Q1), 2001-2005 (Q2), 2006-2010 (Q3), 2011-2015 (Q4), se ordenaron } \\
\text { según los } 20 \text { municipios con las tasas corregidas de homicidio más elevadas en el último quinquenio (Q4). }\end{array}$} \\
\hline
\end{tabular}


El estado de Bahía tuvo el $25,5 \%$ de subestimación total en los datos obtenidos a partir del SIM, en el período de 2011-2015 (Q4). La región oeste, a pesar de presentar la menor tasa de homicidios corregida entre las macrorregiones de salud del estado $(12,2 / 100.000$ habitantes), en el Q4 fue la que sufrió el mayor efecto del proceso de corrección, con una variación del $124,1 \%$ entre el número de homicidios sin corrección y el total de homicidios luego de la segunda corrección. Uno de los municipios de esa región, Bom Jesus da Lapa, llegó a duplicar el número de homicidios con las correcciones (Tabla 3).

Tabla 3. Número y tasa de homicidio corregidos, en los 20 municipios con mayores tasas de homicidio por macrorregiones. Estado de Bahía, Brasil, 2011-2015.

\begin{tabular}{|c|c|c|c|c|c|}
\hline \multirow[b]{2}{*}{ Municipio/macrorregión } & \multicolumn{3}{|c|}{ Número de homicidios } & \multirow{2}{*}{$\begin{array}{l}\text { Factor de } \\
\text { corrección }{ }^{d}\end{array}$} & \multirow{2}{*}{$\begin{array}{c}\text { Tasa } \\
\text { corregidae }\end{array}$} \\
\hline & $\mathrm{SIM}^{\mathrm{a}}$ & $\begin{array}{l}\text { Primeira } \\
\text { corrección }\end{array}$ & $\begin{array}{l}\text { Segunda } \\
\text { corrección }\end{array}$ & & \\
\hline Bahía & 27.885 & 31.032 & 34.983 & - & 31,1 \\
\hline Centro-este & 2.723 & 3.721 & 4.180 & - & 24,2 \\
\hline Amélia Rodrigues & 80 & 89 & 108 & 1,21 & 83,7 \\
\hline Terra Nova & 39 & 41 & 49 & 1,22 & 73,8 \\
\hline Feira de Santana & 1.297 & 1.911 & 2.045 & 1,07 & 69,2 \\
\hline Centro-norte & 998 & 1.054 & 1.253 & - & 26,4 \\
\hline Irecê & 184 & 186 & 209 & 1,12 & 59,1 \\
\hline Presidente Dutra & 32 & 32 & 39 & 1,22 & 54,0 \\
\hline Capim Grosso & 51 & 57 & 69 & 1,21 & 48,8 \\
\hline Extremo sur & 2.595 & 2.837 & 3.263 & - & 66,6 \\
\hline Santa Cruz Cabrália & 147 & 147 & 178 & 1,21 & 128,2 \\
\hline Itabela & 123 & 142 & 172 & 1,21 & 113,3 \\
\hline Porto Seguro & 645 & 656 & 735 & 1,12 & 106,8 \\
\hline Este & 12.313 & 13.397 & 14.707 & - & 47,1 \\
\hline Pojuca & 186 & 193 & 233 & 1,21 & 129,8 \\
\hline Lauro de Freitas & 879 & 947 & 1.061 & 1,12 & 117,7 \\
\hline Simões Filho & 592 & 647 & 725 & 1,12 & 114,3 \\
\hline Nordeste & 1.538 & 1.634 & 1.922 & - & 34,2 \\
\hline Alagoinhas & 483 & 512 & 573 & 1,12 & 76,7 \\
\hline Esplanada & 99 & 103 & 125 & 1,21 & 70,8 \\
\hline Catu & 154 & 160 & 180 & 1,12 & 66,6 \\
\hline Norte & 1.502 & 1.558 & 1.796 & - & 28,3 \\
\hline Juazeiro & 534 & 549 & 615 & 1,12 & 58,5 \\
\hline Rodelas & 20 & 20 & 24 & 1,22 & 57,2 \\
\hline Paulo Afonso & 254 & 266 & 298 & 1,12 & 51,7 \\
\hline Oeste & 295 & 563 & 661 & - & 12,2 \\
\hline São Félix do Coribe & 26 & 34 & 41 & 1,22 & 57,6 \\
\hline Santa Maria da Vitória & 34 & 53 & 64 & 1,21 & 31,3 \\
\hline Bom Jesus da Lapa & 46 & 87 & 97 & 1,12 & 29,0 \\
\hline Sudoeste & 1.947 & 2.050 & 2.335 & - & 15,8 \\
\hline Vitória da Conquista & 845 & 886 & 948 & 1,07 & 57,7 \\
\hline Itororó & 41 & 42 & 51 & 1,22 & 50,0 \\
\hline Itambé & 39 & 41 & 50 & 1,21 & 43,0 \\
\hline Sur & 3.974 & 4.218 & 4.866 & - & 45,1 \\
\hline Santa Luzia & 59 & 67 & 82 & 1,22 & 121,4 \\
\hline Valença & 358 & 367 & 411 & 1,12 & 87,6 \\
\hline Itabuna & 816 & 859 & 919 & 1,07 & 86,5 \\
\hline
\end{tabular}


En la Tabla 3, al ordenar el número y las tasas de homicidio de acuerdo con las macrorregiones de salud, se verifica la aparición de otros municipios importantes en términos poblacionales y el número absoluto de homicidios. Feira de Santana, Lauro de Freitas, Vitória da Conquista e Itabuna fueron los que presentaron los mayores números de homicidios después de las etapas de corrección. En Feira de Santana, municipio de la región metropolitana del estado, situado en la macrorregión de salud centro-este, los homicidios pasaron de 1.297 (datos del SIM), a 2.045 casos (luego de la corrección), una subestimación del $57,7 \%$ en el Q4.

\section{DISCUSIÓN}

Los índices de homicidio en Bahía y en sus municipios, según los resultados de este estudio, tuvieron incrementos relevantes a lo largo de las dos décadas analizadas. El ajuste en el total de defunciones, luego de las correcciones, alcanzó hasta un $100 \%$ en uno de los municipios bahiano, entre 1996 y 2015. Al respecto, un estudio señaló que hubo un crecimiento del $242,1 \%$ en el número de homicidios en Bahía, en el período de 2002 a $2012^{(4)}$. Por lo tanto, con el objetivo de mejorar su calidad, el ajuste efectuado en la información sobre causas externas y homicidios a partir de los eventos de intención indeterminada, da cuenta solo de una parte de los crecientes índices de homicidio en el estado ${ }^{(4)}$.

Un ejemplo que ilustra esta última cuestión está presente en los hallazgos de este estudio, que encontró mayor subestimación de defunciones en los datos referentes al quinquenio 2001-2005. En este período, de acuerdo con Cerqueira ${ }^{(7)}$, hubo también una mayor proporción de defunciones con intención indeterminada. Este autor afirma que, desde 2007 a 2010, Bahía presentó la mayor tasa de este tipo de muertes $(12,9 / 100.000$ habitantes) entre las unidades federativas, quedando debajo del estado del Río de Janeiro $(18,1 / 100.000$ habitantes). Destaca, además, que Bahía fue el estado brasileño que presentó la mayor diferencia entre el crecimiento de las tasas de homicidios oficiales y estimadas en el período 1996-2010, o sea, cuando se compararon las tasas calculadas con base en los datos del SIM, se corrigieron las defunciones y se consideró el grupo de los eventos con intención indeterminada ${ }^{(7)}$.

En el período analizado en este trabajo, la subestimación de defunciones por homicidios en Bahía fue de más de 20.000 casos, lo que representó casi $1 / 3$ de los homicidios registrados oficialmente en ese estado por el Sistema de Información sobre Mortalidad. Según el Ministerio de Justicia ${ }^{(5)}$, Bahía llegó a registrar la sexta mayor tasa de homicidio en el país, 36,0 por 100.000 habitantes en el año 2014. En algunos municipios del estado las tasas son aún más elevadas: Porto Seguro (86,5/100.000), Simões Filho (84,3/100.000), Camaçari $(77,1 / 100.000)$, Lauro de Freitas $(77,1 / 100.000)$, Itabuna $(69,0 / 100.000)$, Ilhéus (62,0/100.000), Feira de Santana $(54,9 / 100.000)$, Juazeiro $(49,4 / 100.000)$, Vitória da Conquista $(47,9 / 100.000)$ y Salvador $(43,6 / 100.000)$.

El ordenamiento de las defunciones y tasas de homicidio de acuerdo con los mayores índices presentados en el último quinquenio (Q4) se corroboró tanto con estudios realizados localmente que tenían el estado de Bahía y sus municipios como únicas unidades de análisis, como con estudios de bases nacionales $s^{(4,14,15)}$. El método de ordenamiento posibilitó considerar los años (quinquenios) anteriores y la evolución de las defunciones en el tiempo, como también focalizar el análisis a los datos más actuales. De los 417 municipios del estado de Bahía, no son pocos los que se destacan en el escenario nacional. Entre las ciudades con más de 10.000 habitantes, de las diez con las mayores tasas de homicidio en 2012, la mitad eran de Bahía y de acuerdo con el Mapa de la violencia de $2014^{(4)}$, Mata de São João $(149,3)$ ocupó la segunda posición; Simões Filho $(131,0)$, la tercera; Ibirapitanga $(123,4)$, la sexta; Itaparica $(119,1)$, la octava; y Porto Seguro $(115,5)$, la décima posición en el ranking del país.

Otros estudios ${ }^{(14,15,16)}$ destacan que la intensificación de este fenómeno en ciertas 
localidades del estado se potencia, probablemente, por la expansión de la actividad turística y recuperación de la economía relacionada con la producción de cacao, que atrae migrantes y genera oportunidades, pero también aumenta los problemas sociales y conflictos. A pesar de que el riesgo de muerte por homicidio se encuentre vastamente diseminado por todo el territorio bahiano, en los municipios de la franja costera, la región metropolitana (este) y en el triángulo formado por Porto Seguro, Vitória da Conquista e Itabuna, el fenómeno cobra perfiles alarmantes.

Un aspecto a destacar de este estudio es considerar el tamaño poblacional de los municipios, tanto para la aplicación del factor de corrección, como en la descripción y ordenamiento de los municipios. De los 20 municipios destacados, Itabuna y Camaçari fueron los únicos clasificados como de tamaño grande (más de 200.000 habitantes), en el ordenamiento de las tasas quinquenales de homicidio. Hubo seis municipios de tamaño pequeño/medio, ocho de tamaño medio/grande y cuatro de tamaño pequeño. Como en un total de 20 municipios, ocho son de tamaño medio/grande (50.001 a 200.000 habitantes), esto puede sugerir que en esas ciudades existe más desigualdad socioeconómica que en aquellas de tamaño pequeño/medio y pequeño. Estos municipios de tamaño medio tienden a tener una economía activa más concentrada, además de no garantizar los servicios y las oportunidades existentes en los municipios de gran tamaño y en las metrópolis; pero, probablemente, por ocupar ese "lugar del medio" sufren los efectos de las desigualdades y heterogeneidades locales ${ }^{(17)}$.

Esta hipótesis es corroborada por un estudio ecológico de los municipios brasileiros que reveló incrementos temporales significativos de las tasas cuatrienales de homicidio, sobre todo en municipios de medio tamaño, caracterizados por menos desarroIlo socioeconómico y mayor desigualdad económica ${ }^{(17,18)}$. Aunque los municipios de mayor tamaño poblacional y con mayor urbanización presenten tasas de homicidio más elevadas, los mayores riesgos de homicidio se observaron en municipios con mayores desigualdades sociales y con niveles de ingresos y pobreza intermedios.

Si bien muchos otros factores necesitan ser considerados para comprender este "sube y baja" en la distribución de los índices de homicidio entre las ciudades de una determinada región y los porqués del aumento del homicidio en algunos lugares y su disminución en otros ${ }^{(15,17,19)}$, el foco del presente estudio y de sus hallazgos no es discutir sobre aspectos referentes a las desigualdades y sobre el desarrollo económico de los municipios, sino sobre la posibilidad de la corrección y estimación de tasas de mortalidad más fidedignas.

Más allá de que hayan sido adoptadas medidas para reducir la inestabilidad de las tasas y para corregir el número de defunciones, se considera que la subestimación persiste a través de los años y municipios estudiados, sobre todo en aquellos de menor tamaño y que no poseen un Instituto Médico Legal o Servicio de Verificación de Defunciones $^{(10,20,21)}$. Algunas propuestas que se podrían realizar se refieren a la baja informatización en muchos municipios pequeños, la falta de calificación de los profesionales y la sobrecarga de trabajo, no solo del perito legista en la posnecropsia, sino también de los médicos de instituciones hospitalarias (principalmente, emergencias) y demás servicios de salud, que son responsables de identificar la muerte violenta o sospechosa y derivar el cadáver/cuerpo al Instituto Médico Legal y al Servicio de Verificación de Defunciones ${ }^{(20,21)}$.

Otros factores que también contribuyen a la subestimación son los cementerios clandestinos y el número de personas desaparecidas, que pueden encubrir casos de homicidio e intervenciones legales/muertes derivadas de acciones de la policía o de otros agentes de la ley/militares en servicio ${ }^{(20,21,22)}$. Además, el aumento de la cobertura de las defunciones en Brasil, sobre todo a partir de 2000, ocurrió con el proceso de municipalización, que incorporó y responsabilizó a los municipios por los procedimientos y el procesamiento de los datos de mortalidad en el ámbito nacional. 
Datos de 1997 mostraban una cobertura del $56,2 \%$ en el nordeste, siendo que la media de Brasil era del $80 \%{ }^{(20,21)}$.

Al analizar la calidad de las estadísticas vitales brasileñas, en la temática de los homicidios, Mello Jorge et al. ${ }^{(11)}$ observaron cambios en el panorama de las muertes por causas externas y señalaron que, en algunas áreas del país, en el año 2000, el total de homicidios presentó un aumento de cinco veces lo declarado en las bases oficiales, luego de una búsqueda activa de información y en las fuentes primarias del Instituto Médico Legal, esclareciendo la causa básica de muerte, no transcrita en los certificados de defunción.

Se destaca que la imposibilidad de integrar sistemas de información o bases de datos secundarios sobre la violencia en Brasil y en los estados como, por ejemplo, datos del sector de seguridad pública y salud, inviabilizan (o, por el menos, dificultan mucho) la posibilidad de complementariedad de los sistemas como el SIM, en la perspectiva de reducir la subestimación de las defunciones ${ }^{(23)}$.

Otra limitación se refiere a la cobertura de la información sobre mortalidad en Bahía, a partir de los registros del SIM. De acuerdo con el Ministerio de Salud, en el período $1996-2013$, pasó del $62,87 \%$ al $88,75 \%$. Aun siendo una buena cobertura, está por debajo de la media nacional que fue del $94,9 \%{ }^{(3)}$, en ese último año. En el presente estudio, con el propósito de ajustar la subestimación de la cobertura, se aplicaron factores de corrección que remiten a un estudio sobre el año $2008^{(10)}$, mientras las tasas se corrigieron en el período 1996-2015. Esto puede dar lugar a que, en los años de ese período que se alejan más de 2008, como los del primer quinquenio 1996-2000, los factores de corrección, posiblemente, den estimaciones más imprecisas, porque tanto la cobertura como la calidad de la información serían peores, comparadas con los años más recientes.

Aun así, el presente estudio mostró que es posible dar cuenta del subregistro de los datos oficiales y buscar estimaciones de homicidio más fidedignas ${ }^{(20,21)}$ en determinadas localidades y en un determinado período de tiempo. Estas herramientas son útiles para las investigaciones con metodologías que se proponen discutir tales problemas ${ }^{(8,10)}$.

Se concluye que, en los municipios analizados, luego de los ajustes, hubo un incremento de las defunciones por homicidio en todos los quinquenios, lo que dio como resultado una tasa de mortalidad corregida aún más elevada. Por lo tanto, se cree que estos resultados se aproximan más a las realidades locales.

Asimismo, los resultados que se aproximan poco a lo que realmente sucede, al no considerar las limitaciones de los Sistemas de Información, tanto a nivel de los estados brasileños o a nivel regional y municipal, perjudican la comprensión del fenómeno de las muertes por agresiones -los homicidios- en el contexto nacional. Esto puede confundir la percepción respecto de los riesgos y las áreas de mayor concentración de esos eventos debido a la menor calidad de la información. Por consiguiente, la asignación de recursos y la orientación de las políticas de reducción de homicidios, en tanto cuestión de salud y seguridad pública, pueden estar mal direccionadas al estar basados en información poco fidedignas ${ }^{(20,21)}$. 


\section{REFERENCIAS BIBLIOGRÁFICAS}

1. Minayo MCS. Violência e Saúde. Rio de Janeiro: Editora Fiocruz; 2006.

2. Organização Mundial da Saúde. Relatório Mundial sobre a Prevenção da Violência 2014 [Internet]. São Paulo: Organização Mundial de Saúde; 2015 [citado 12 ago 2017]. Disponible en: https://tinyurl.com/y9wcwfw7.

3. Ministério da Saúde, Departamento de Informática do SUS. Informações de Saúde. Portal da Saúde [Internet]; 2017 [citado 12 ago 2017]. Disponible en: https://tinyurl.com/yavvyvgj.

4. Waiselfisz JJ. Mapa da Violência 2014: Os Jovens do Brasil. Brasília: FLACSO; 2014.

5. Engel CL, (coord.). Diagnóstico dos homicídios no Brasil: subsídios para o Pacto Nacional pela Redução de Homicídios. Brasília DF: Ministério da Justiça; 2015.

6. Mascarenhas MDM, Monteiro RA, Sá NNB, Gonzaga LAA, Neves ACM, Silva DLMMA, Duarte EC, Malta DC. Epidemiologia das causas externas no Brasil: mortalidade por acidentes e violências no período de 2000 a 2009. En: Saúde Brasil 2010: uma análise da situação de saúde e de evidências selecionadas de impacto de ações de vigilância em saúde. Brasília DF: Ministério da Saúde; 2011. p. 225-249.

7. Cerqueira DRC. Mapa de homicídios ocultos no Brasil. Instituto de Pesquisa Econômica Aplicada [Internet]; 2013 [citado 2 agosto 2017]. Disponible en: https://tinyurl.com/yb3qt6uo.

8. Souza ER, Minayo MCS, Silva CMF, Reis AC, Malaquias JV, Veiga JPC, Santos NC, Borges KF, Silva CFR. Análise temporal da mortalidade por causas externas no Brasil: Décadas de 80 e 90. En: Minayo MCS, Souza ER, (orgs.). Violência sob o olhar da saúde: a infrapolítica da contemporaneidade brasileira. Rio de Janeiro: Fiocruz; 2003. p. 83-107.

9. Centro Brasileiro de Classificação de Doenças. Classificação estatística internacional de doenças e problemas relacionados à saúde: décima revisão (CID-10). Departamento de Informática do SUS [Internet]; 2008 [citado 2 ago 2017]. Disponible en: https://tinyurl.com/mgekz8a.

10. Garcia LP, Freitas LRS, Silva GDM, Höfelmann DA. Estimativas corrigidas de feminicídios no Brasil, 2009 a 2011. Revista Panamericana de Salud Pública. 2015;37(4-5):251-257.

11. Jorge MHPM, Laurenti R, Gotlieb SLD. Análise da qualidade das estatísticas vitais brasileiras: a ex- periência de implantação do SIM e do SINASC. Ciência \& Saúde Coletiva. 2007;12(3):643-654.

12. Instituto Brasileiro de Geografia e Estatística. Censo demográfico 2010 [Internet]. Instituto Brasileiro de Geografia e Estatística [citado 20 ago 2017]. Disponible en: https://tinyurl.com/ y88bbvqc.

13. Szwarcwald CL, Morais-Neto OL, Frias PG, Souza JPRB, Escalante JJC, Lima RB, Viola RC. Busca ativa de óbitos e nascimentos no Nordeste e na Amazônia Legal: estimação das coberturas do SIM e do Sinasc nos municípios brasileiros. En: Saúde Brasil 2010: uma análise da situação de saúde e de evidências selecionadas de impacto de ações de vigilância em saúde [Internet]. Brasília DF: Ministério da Saúde; 2011. [citado 26 ago 2017]. p. 79-98. Disponible en: https://tinyurl. com/y7x7o5ez.

14. Andrade LT de, Diniz AMA. A reorganização espacial dos homicídios no Brasil e a tese da interiorização. Revista Brasileira de Estudos de População. 2013;30:S171-S191. doi: 10.1590/ S0102-30982013000400011.

15. Souza TO, Pinto LW, Souza ER. Spatial study of homicide rates in the state of Bahia, Brazil, 19962010. Revista de Saúde Pública. 2014;48(3):468477. doi: 10.1590/S0034-8910.2014048005201.

16. Sousa CAM, Silva CMFP, Souza ER. Determinantes dos homicídios no Estado da Bahia, Brasil, em 2009. Revista Brasileira de Epidemiologia. 2014;17(1):135-146. doi: 10.1590/1415-790X20 $1400010011 \mathrm{ENG}$.

17. Duarte EC, Garcia LP, Freitas LRS, Mansano $\mathrm{NH}$, Monteiro RA, Ramalho WM. Associação ecológica entre características dos municípios e o risco de homicídios em homens adultos de 20-39 anos de idade no Brasil, 1999-2010. Ciência \& Saúde Coletiva. 2012;17(9):2259-68. doi: 10.1590/S1413-81232012000900008.

18. Minayo MCS. Relaciones entre procesos sociales, violencia y calidad de vida. Salud Colectiva. 2005;1(1):69-78. doi: 10.18294/sc.2005.37.

19. Peres MFT, Vicentin D, Nery MB, Lima RS, Souza ER, Cerda M, et al. Queda dos homicídios em São Paulo, Brasil: uma análise descritiva. Revista Panamericana de Salud Pública. 2011;29(1):17-26.

20. Ministério da Saúde, Organização Pan-Americana da Saúde, Fundação Oswaldo Cruz. A experiência brasileira em sistemas de informação em saúde: Produção e disseminação de informações sobre saúde no Brasil [Internet]. Vol. 1. Brasília DF: 
Editora do Ministério da Saúde; 2009 [citado 26 ago 2017]. Disponible en: https://tinyurl.com/oq8ofva.

21. Ministério da Saúde, Organização Pan-Americana da Saúde, Fundação Oswaldo Cruz. A experiência brasileira em sistemas de informação em saúde: Falando sobre os sistemas de informação em saúde no Brasil [Internet]. Vol. 2. Brasília: Editora do Ministério da Saúde; 2009 [citado 26 ago 2017]. Disponible en: https://tinyurl.com/ y8wjpuwp.
22. Cardoso FLMG, Cecchetto FR, Corrêa JS, Souza TO. Homicídios no Rio de Janeiro, Brasil: uma análise da violência letal. Ciência \& Saúde Coletiva. 2016;21(4):1277-1288. doi: 10.1590/141381232015214.14712015.

23. Silva CF, Leite AJM, Almeida NMGS. Linkage entre bancos de dados de nascidos vivos e óbitos infantis em município do Nordeste do Brasil: qualidade dos sistemas de informação. Cadernos de Saúde Pública. 2009;25(7):1552-1558.

\section{FORMA DE CITAR}

Souza TO, Pinto LW, Souza ER. Muertes ocultas: estimaciones corregidas de homicidios en Bahía, Brasil, 1996-2015. Salud Colectiva. 2019;15:e2106. doi: 10.18294/sc.2019.1639.

Recibido: 13 de noviembre de 2017 | Versión final: 10 de septiembre de 2018 | Aprobado: 21 de septiembre de 2018

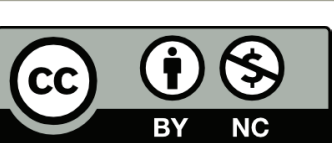

Esta obra está bajo una licencia de Creative Commons Reconocimiento-NoComercial 4.0

Internacional. Reconocimiento - Permite copiar, distribuir y comunicar públicamente la obra.

A cambio, se debe reconocer y citar al autor original. No Comercial - Esta obra no puede ser

utilizada con finalidades comerciales, a menos que se obtenga el permiso.

Este artículo fue traducido del portugués por Viviana Martinovich.

http://dx.doi.org/10.18294/sc.2019.1639 